\title{
PARAMETER SENSITIVITY MAPS OF SURFACE GEOELECTRIC ARRAYS II. NONLINEAR AND FOCUSSED ARRAYS
}

\author{
S Szalai and L Szarka \\ Geodetic and Geophysical Research Institute of the Hungarian Academy of Sciences, \\ POB 5, H-9401 Sopron, Hungary, e-mail: szalai@ggki.hu; szarka@ggki.hu
}

[Manuscript received January 15, 2008; accepted March 17, 2008]

\begin{abstract}
In this paper we present, at first time in a geophysical journal, parameter sensitivity maps of nonlinear and focussed electrode arrays. We present them as anomalies due to electric dipoles forming on opposing surfaces of an elementary cube within the subsurface at three different depths, and not only the total effect of the dipole, but also of its components are shown. Parameter sensitivity maps of non-linear arrays, compared to those of linear arrays, have in general 1 . more equal sensitivity values in $\mathrm{x}$ and $\mathrm{y}$ directions, 2. more chances for antisymmetry axes, 3. smoother lateral distribution of sensitivity values. We recommend a systematic use of parameter sensitivity maps in geoelectric prospecting, both in planning and interpretation of field measurements.
\end{abstract}

Keywords: geoelectric arrays; parameter sensitivity; surface geoelectric methods

\section{Introduction}

In the first part of the paper (Szalai and Szarka 2008) we discussed the development of parameter sensitivity studies and techniques of calculation of parameter sensitivity maps. Parameter sensitivity maps were presented for 14 linear arrays. Although some of the results had already been known, this paper, together with the accompanying one (Szalai and Szarka 2008) is their first complete presentation of parameter sensitivity maps of surface geoelectric arrays. In this second part, parameter sensitivity maps of 7 nonlinear and 3 focussed arrays are discussed. There are more nonlinear and focussed arrays, but not all of them dispose with parameter sensitivity maps. 
Table I. Electrode co-ordinates of nonlinear and focussed arrays for the computation of parameter sensitivity maps

\begin{tabular}{|c|c|c|c|c|c|c|c|c|c|c|c|}
\hline \multirow{2}{*}{$\begin{array}{l}\text { Number of } \\
\text { electrodes }\end{array}$} & \multirow{2}{*}{$\begin{array}{l}\text { Type of } \\
\text { the array }\end{array}$} & \multirow{2}{*}{$\begin{array}{l}\text { Number of } \\
\text { the array }\end{array}$} & \multirow{2}{*}{$\begin{array}{l}\text { Name of } \\
\text { the array }\end{array}$} & \multicolumn{2}{|c|}{$\mathrm{A}$} & \multicolumn{2}{|c|}{$\mathrm{B}$} & \multicolumn{2}{|c|}{$\mathrm{M}$} & \multicolumn{2}{|c|}{$\mathrm{N}$} \\
\hline & & & & $\mathrm{x}$ & $\mathrm{y}$ & $\mathrm{x}$ & $\mathrm{y}$ & $\mathrm{x}$ & $\mathrm{y}$ & $\mathrm{x}$ & $\mathrm{y}$ \\
\hline \multirow{7}{*}{$\begin{array}{l}\text { Four } \\
\text { electrodes }\end{array}$} & \multirow{7}{*}{$\begin{array}{l}\text { Nonlinear } \\
\text { arrays }\end{array}$} & 15 & $\begin{array}{l}\text { Dipole } \\
\text { equatorial }\end{array}$ & 0 & 0.05 & 0 & -0.05 & 1 & 0.05 & 1 & -0.05 \\
\hline & & 16 & $\begin{array}{l}\text { Dipole axial } \\
\text { null }\end{array}$ & 0 & 0 & 0.1 & 0 & 1 & 0.05 & 1 & -0.05 \\
\hline & & 17 & $\begin{array}{l}\text { Dipole } \\
\text { parallel } 54^{\circ}\end{array}$ & 0 & 0 & 0.0058 & -.0081 & 1 & 0 & 1.0058 & -.0081 \\
\hline & & 18 & \multirow{3}{*}{$\begin{array}{l}\text { Square- } \alpha \\
\text { Square- } \gamma \\
\text { Schlumberger } \\
\text { null }\end{array}$} & 0.2 & -0.3 & 0.8 & -0.3 & 0.2 & 0.3 & 0.8 & 0.3 \\
\hline & & 19 & & 0 & 0 & 1 & 0 & 0.5 & 0.5 & 0.5 & -0.5 \\
\hline & & 20 & & 0 & 0 & 1 & 0 & 0.5 & 0.125 & 0.5 & -0.125 \\
\hline & & 21 & $\begin{array}{l}\text { Three-electrode } \\
\text { null }\end{array}$ & 0 & 0 & $\inf$ & 0 & 1 & 0.125 & 1 & -0.125 \\
\hline More than & Linear & 22 & Unipole- $\alpha$ & 0 & 0 & $1^{*}$ & 0 & 0.33 & 0 & 0.67 & 0 \\
\hline four & arrays & 23 & Unipole- $\beta$ & 0 & 0 & $0.33^{*}$ & 0 & 0.67 & 0 & 1 & 0 \\
\hline electrodes & & 24 & Unipole- $\gamma$ & 0 & 0 & $0.67^{*}$ & 0 & 0.33 & 0 & 1 & 0 \\
\hline
\end{tabular}

${ }^{*}$ Means identical signs (current directions) of the two current electrodes. The numbering expresses a continuity with linear, non-focussed arrays (Szalai and Szarka 2008)

\section{Parameter sensitivity maps of surface nonlinear and focussed arrays}

In Figures 1-10 parameter sensitivity maps of seven nonlinear and three focussed arrays are shown. The array names and the corresponding electrode co-ordinates are listed in Table I.

In Fig. 1, the parameter sensitivity maps of the dipole-equatorial array is shown. Only the total effect was published before (Hursán 1996). We present parameter sensitivity maps of two further nonlinear dipole-dipole arrays: the dipole axial null array (in Fig. 2, Szalai et al. 2002), and the $54^{\circ}$ parallel dipole array (Fig. 3). In Figs 4 and 5 two variants of the square array are shown. In Fig. 6. the Schlumberger null array (Szalai et al. 2002), in Fig. 7 the three-electrode null array (Szalai et al. 2002) are shown. In Figs $8-10$ three focussed arrays: the unipole- $\alpha,-\beta$ and $-\gamma$ arrays are presented.

\section{Main features of parameter sensitivity maps}

In case of nongradient-like nonlinear arrays (Figs 4-5) and two dipole-dipole nonlinear arrays (Figs 1,3) the role of $y$ component becomes significant. It may be even more significant than the role of $x$ component! In case of linear focussed arrays (Figs 8-10) the $x$ component remains dominant, but here the role of the $z$ component will be larger than in other cases.

In most of the maps (in any depth and with any components) there are also negative zones. The zero isolines, separating the zones of positive and negative parameter sensitivity values, have characteristic shapes. For example in case of most of the null arrays (Figs 2, 5, 6, 7, 8) they are linear. 

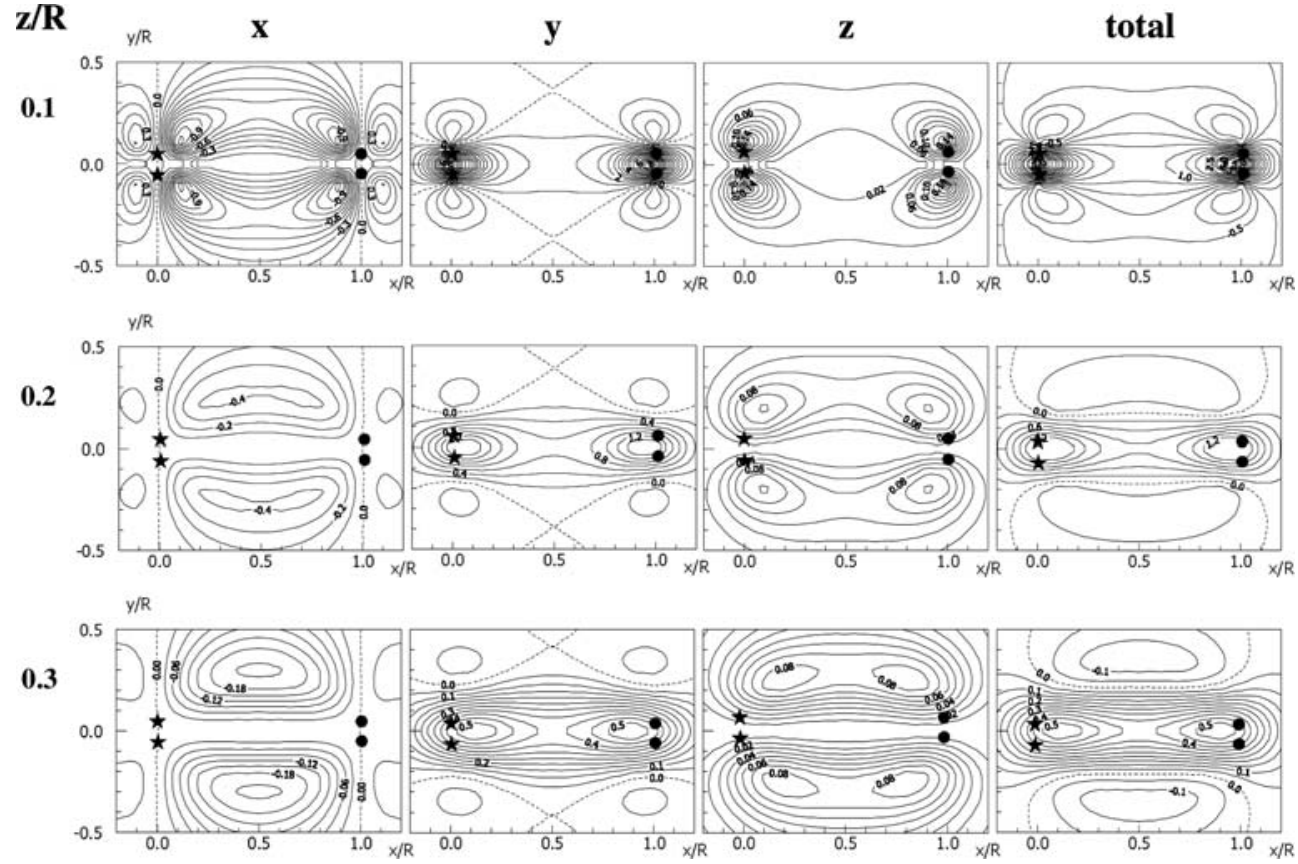

Fig. 1. Parameter-sensitivity map series of the dipole-equatorial array (No. 15 in Table I), with the current electrode (stars) and potential electrode (circles) positions. $x, y, z$ components illustrate the effect of electrical charges accumulated at the corresponding opposite cube faces, while "total" means their superposed effect. The maps were made at three various depths/characteristic length values $(0.1,0.2$ and 0.3$)$

The unipole arrays (in Figs 8-10) have one symmetry axis, while the dipole equatorial (Fig. 1) and the square- $\alpha$ (Fig. 4) arrays have two symmetry axes. The dipole axial null array (Fig. 2), three three-electrode null (Fig. 7) and the unipole- $\alpha$ (Fig. 8) arrays have one antisymmetry axis, while the square- $\gamma$ (Fig. 5) and the Schlumberger null (Fig. 6) arrays have two antisymmetry axes. They provide ideal vectorial information about the subsurface. The symmetry properties follow from the original symmetry properties of the arrays. The antisymmetry axes indicate that these arrays are unable to detect certain types of subsurface bodies in certain positions (Szalai et al. 2002).

The lateral distribution of sensitivity values is in general (with exception of dipole-dipole arrays) smoother than in case of the most linear arrays. This feature is due to larger lateral interception of the electrodes. 
$\mathbf{z} / \mathbf{R}$
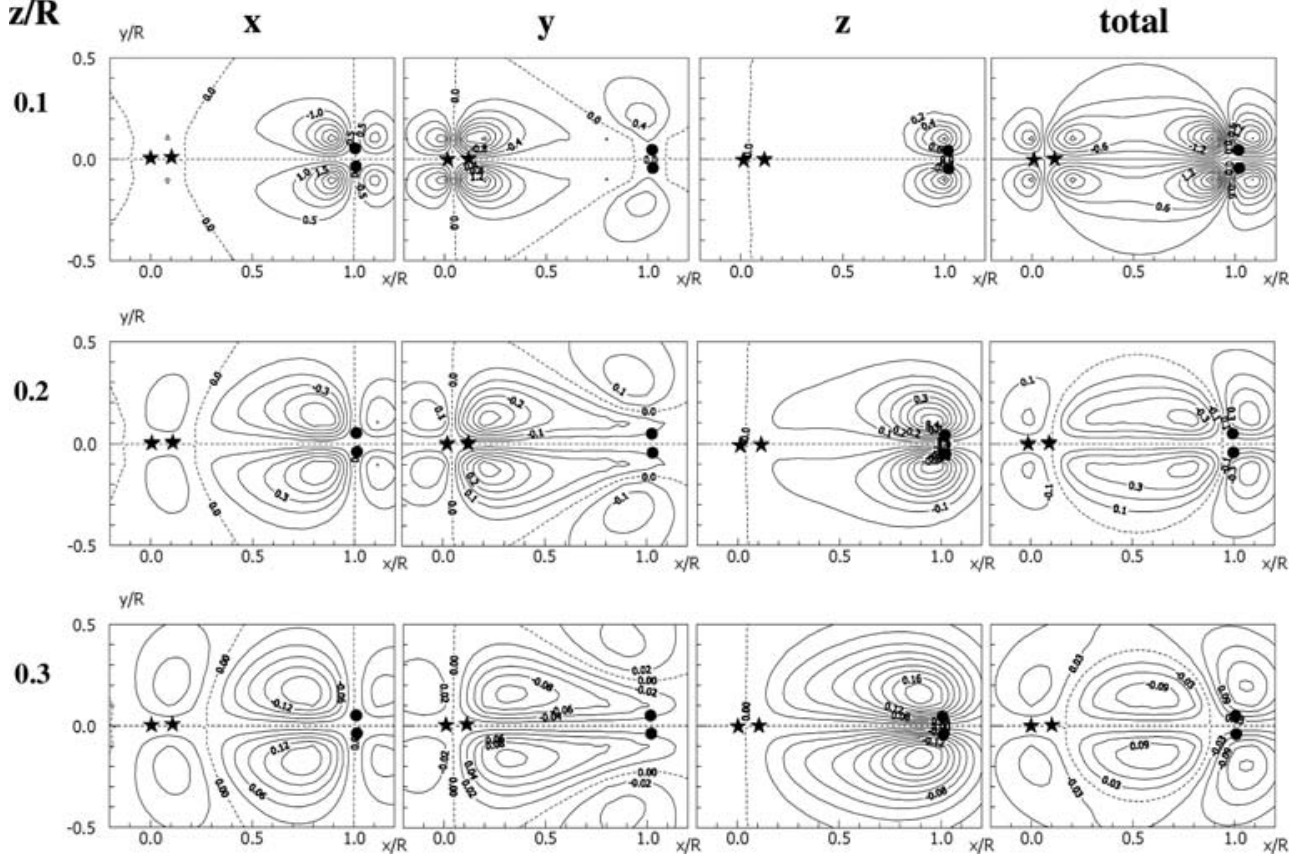

Fig. 2. Parameter-sensitivity map series of the dipole-axial null array (No. 16 in Table I). Notations are the same as in Fig. 1

\section{Conclusions}

We have presented parameter sensitivity maps of altogether 10 nonlinear and focussed geoelectric arrays. They are also results of simple analytical formula, as the parameter sensitivity maps of the 14 linear arrays, presented in the first part of this paper (Szalai and Szarka 2008). The parameter sensitivity values (namely their response to an elementary body in $\mathrm{x}, \mathrm{y}, \mathrm{z}$ position) can be now directly intercompared for $14+10$ arrays.

The physical source of anomaly (the electrical charges) at various cube faces can be separately studied. Separating e.g., the electrodes in y direction, the effect of $y$ component, while with focussing the effect of the $z$ component become larger. The first case is favourable to get horizontal vectorial information, the second one is favourable to get vertical information. The antisymmetry features make it possible to apply the pure anomaly technique (Tarkhov 1957).

A detailed study of parameter sensitivity maps helps in understanding the physics of the resulting anomalies and basic features of various arrays. At the same time, we should again underline that it is not necessary that an array having favourable parameter sensitivity maps over the elementary body, will have equally favourable properties over a multidimensional structure. In case of any given largesize multidimensional body the parameter sensitivity is worth studying by the response due to small changes in the parameters of the given model, as it is done Gyulai (1989). 

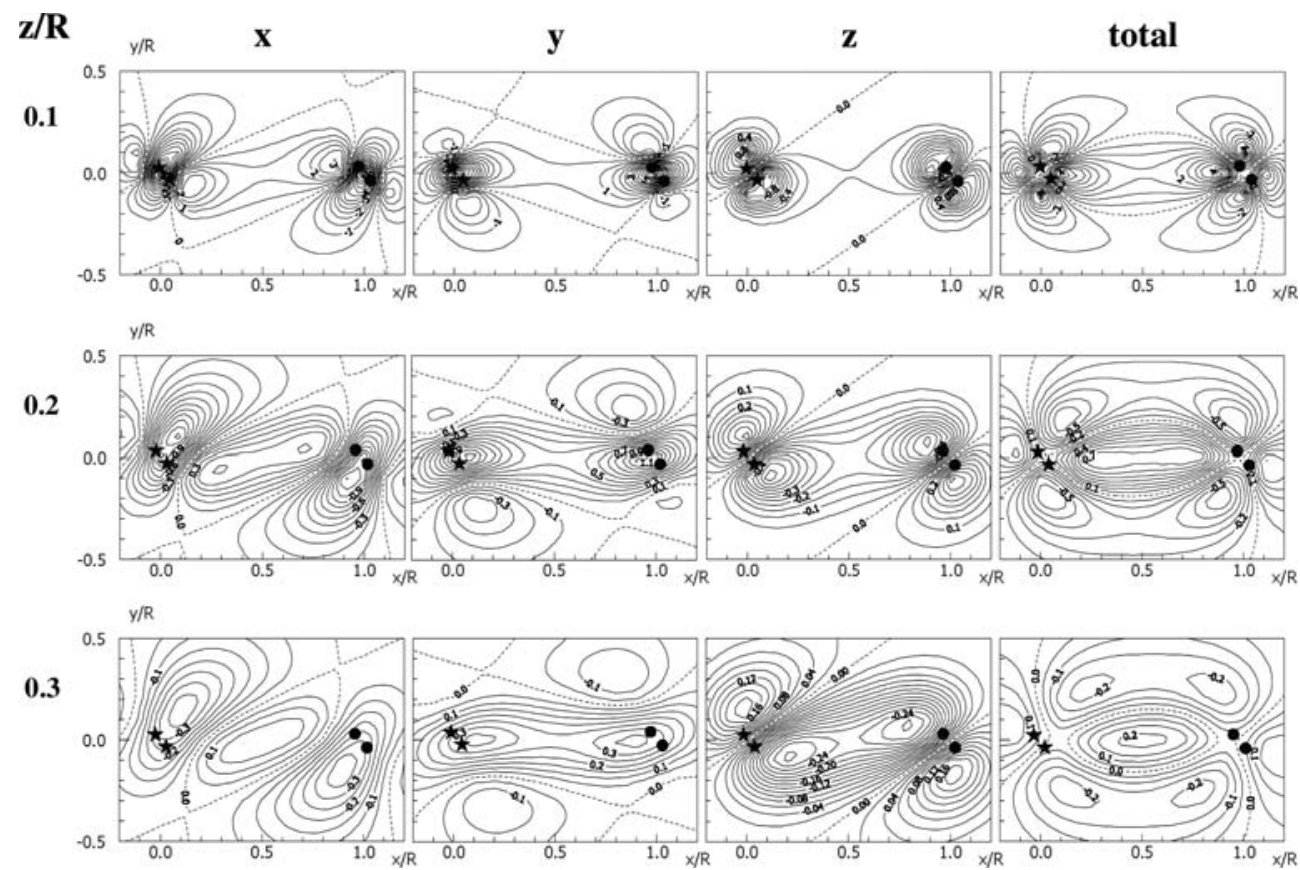

Fig. 3. Parameter-sensitivity map series of the parallel dipole $54^{\circ}$ array (No. 17 in Table I). Notations are the same as in Fig. 1
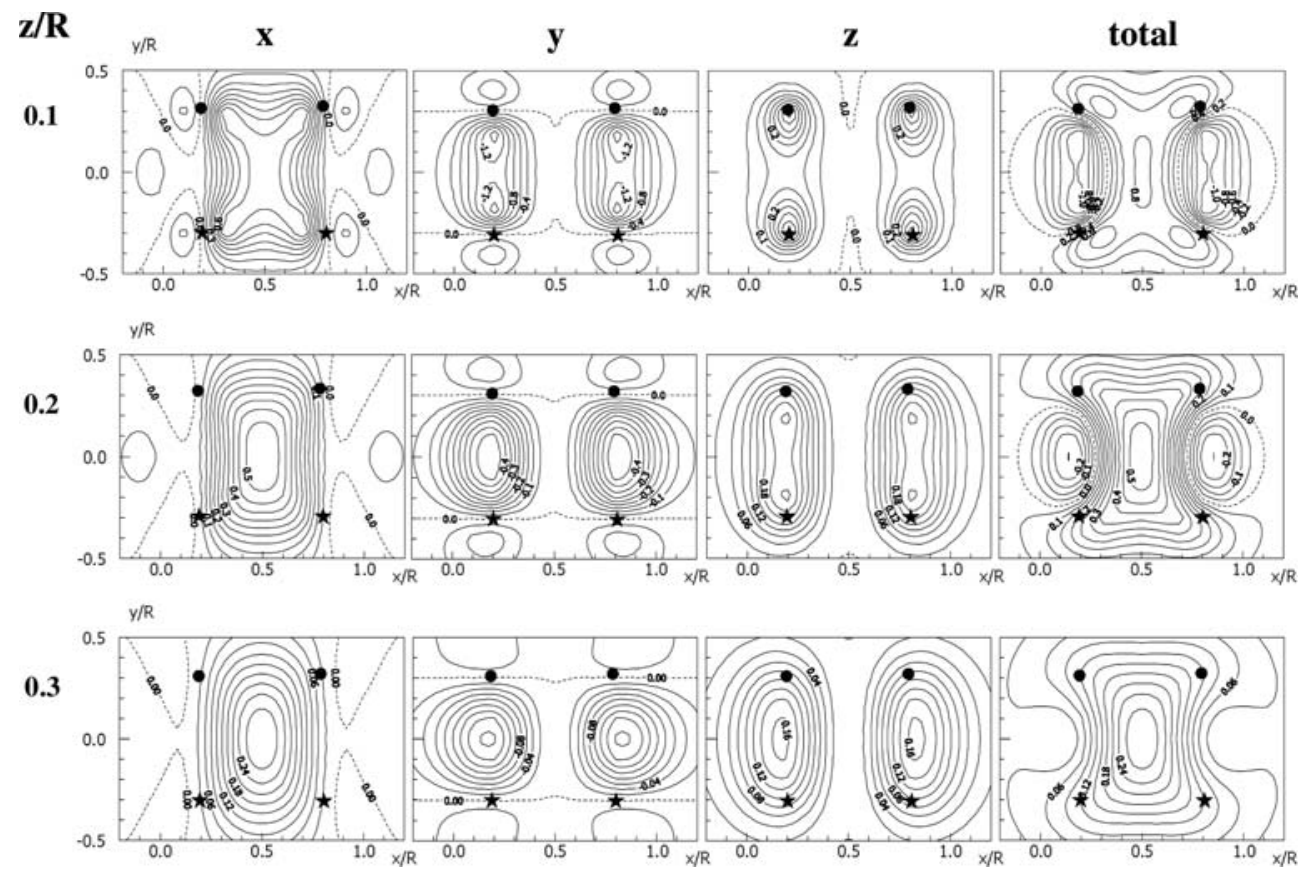

Fig. 4. Parameter-sensitivity map series of the square- $\alpha$ array (No. 18 in Table I). Notations are the same as in Fig. 1 

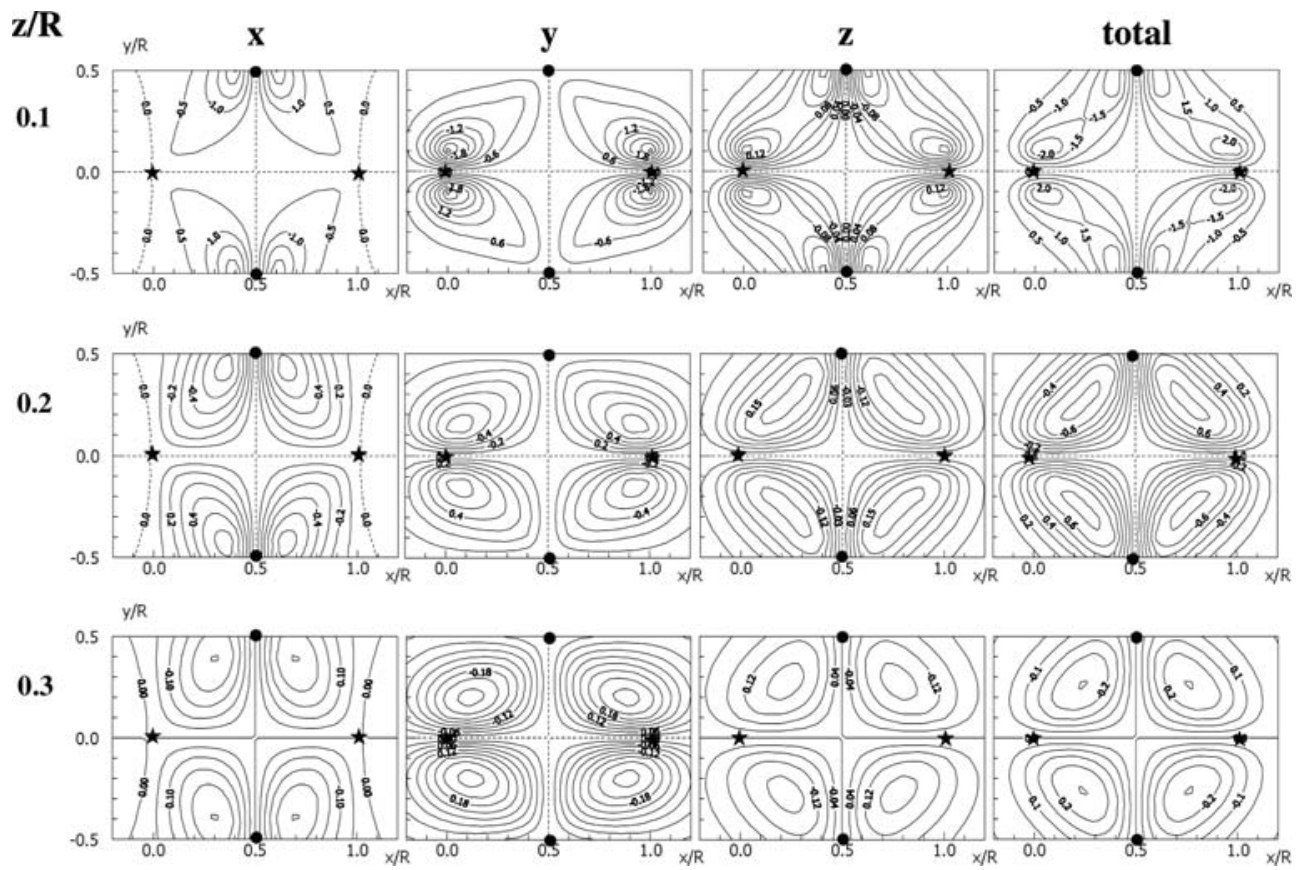

Fig. 5. Parameter-sensitivity map series of the square- $\gamma$ array (No. 19 in Table I). Notations are the same as in Fig. 1
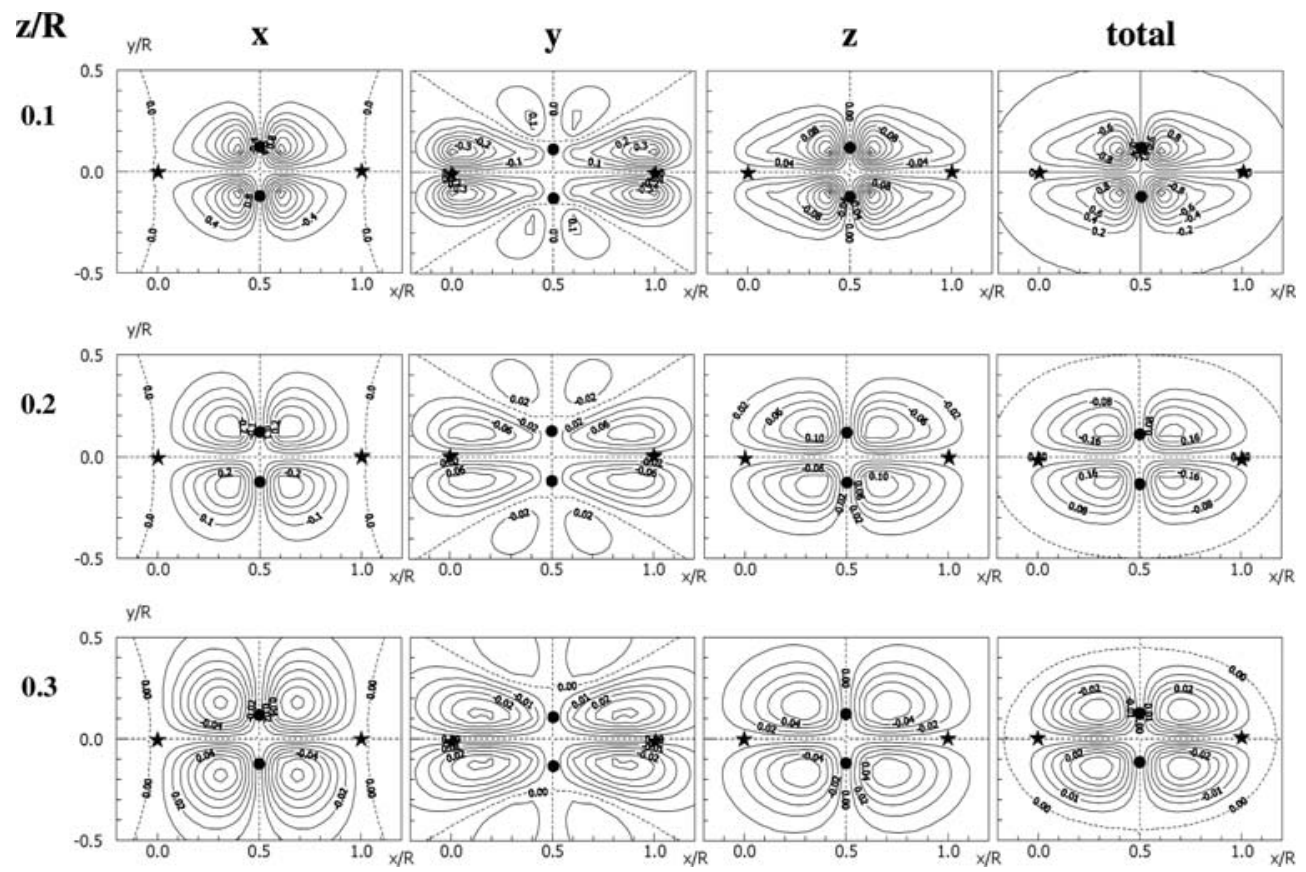

Fig. 6. Parameter-sensitivity map series of the Schlumberger null array (No. 20 in Table I). Notations are the same as in Fig. 1

Acta Geod. Geoph. Hung. 43, 2008 
$\mathbf{z} / \mathbf{R}$ $\mathbf{y} \quad \mathbf{z}$

total

0.1
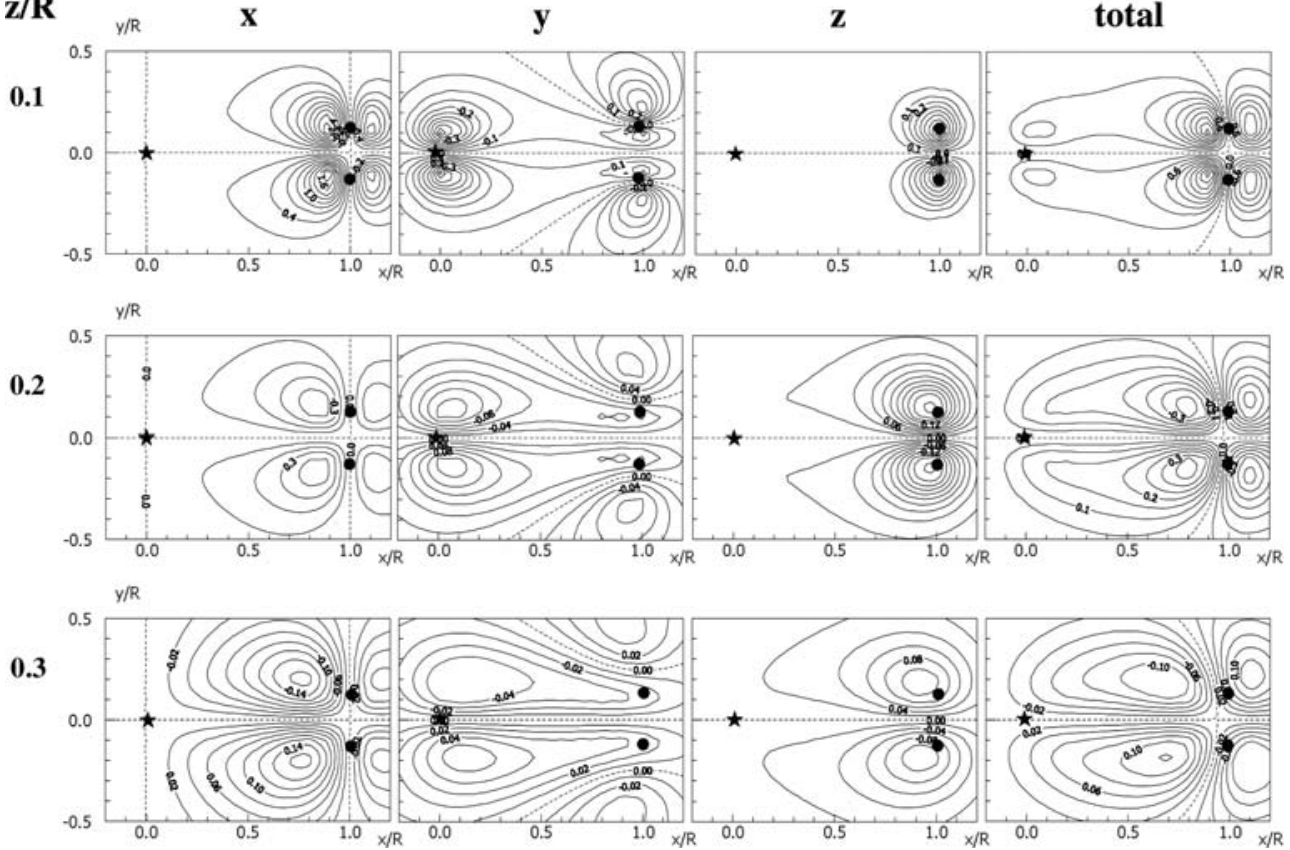

Fig. 7. Parameter-sensitivity map series of the three-electrode null array (No. 21 in Table I). Notations are the same as in Fig. 1
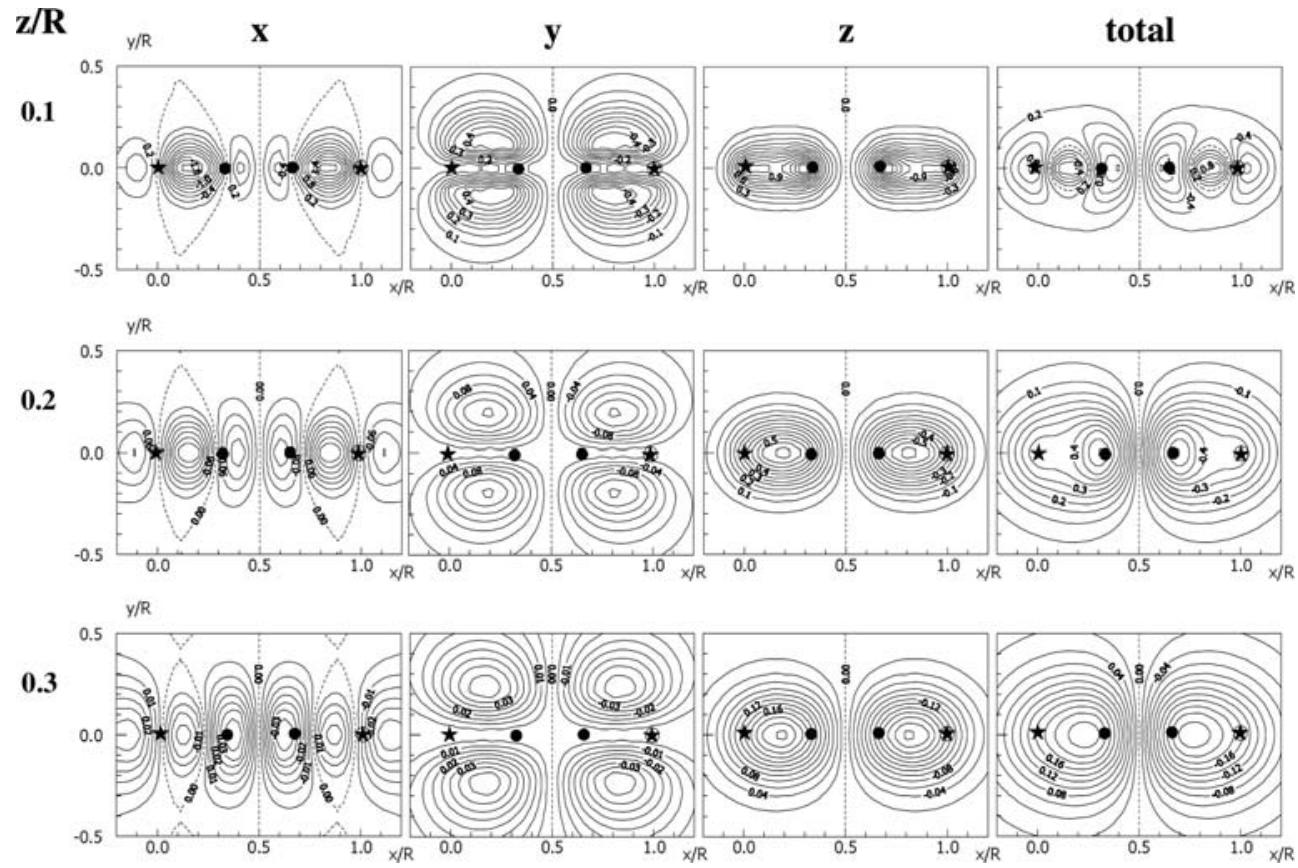

Fig. 8. Parameter-sensitivity map series of the unipole- $\alpha$ array (No. 22 in Table I). Notations are the same as in Fig. 1 

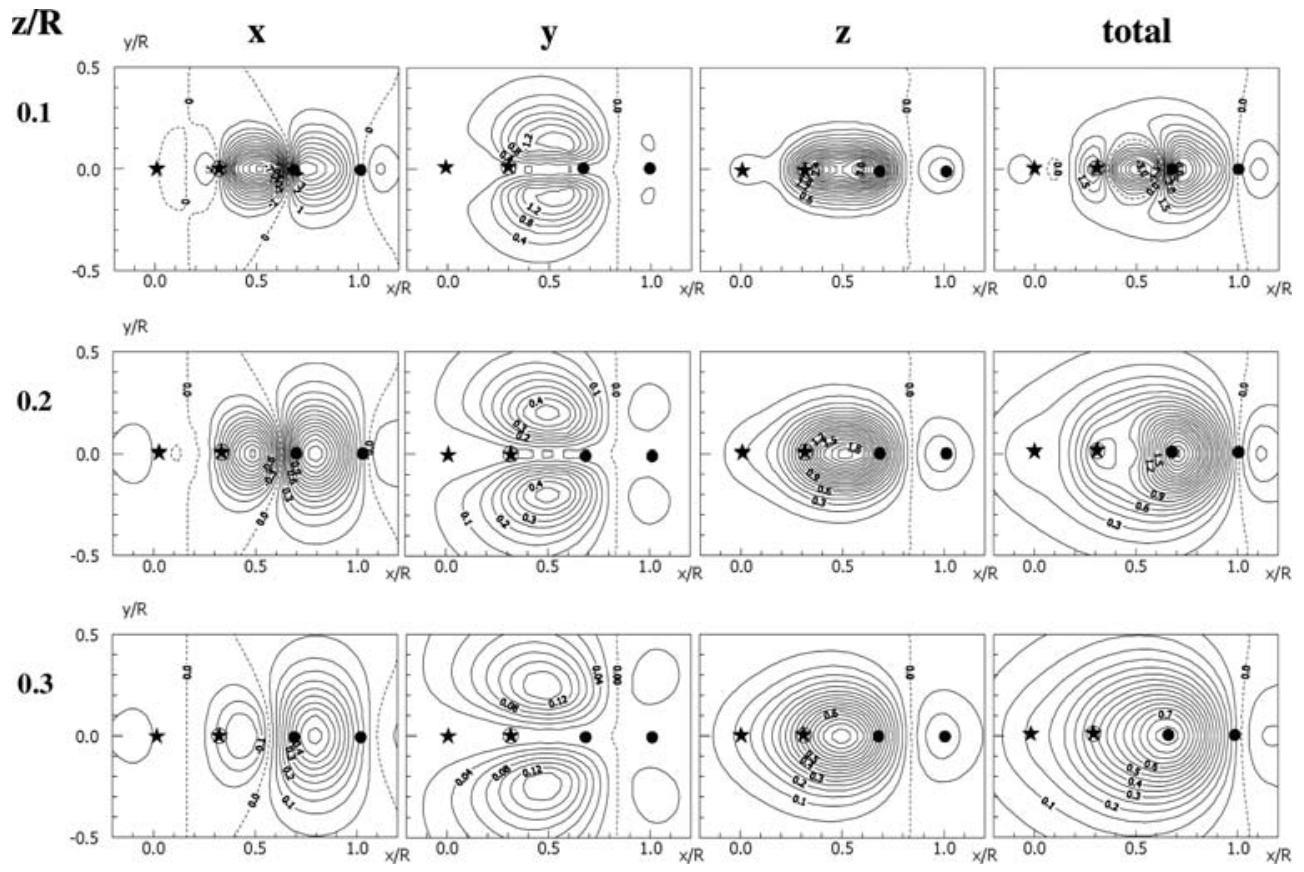

Fig. 9. Parameter-sensitivity map series of the unipole- $\beta$ array (No. 23 in Table I). Notations are the same as in Fig. 1
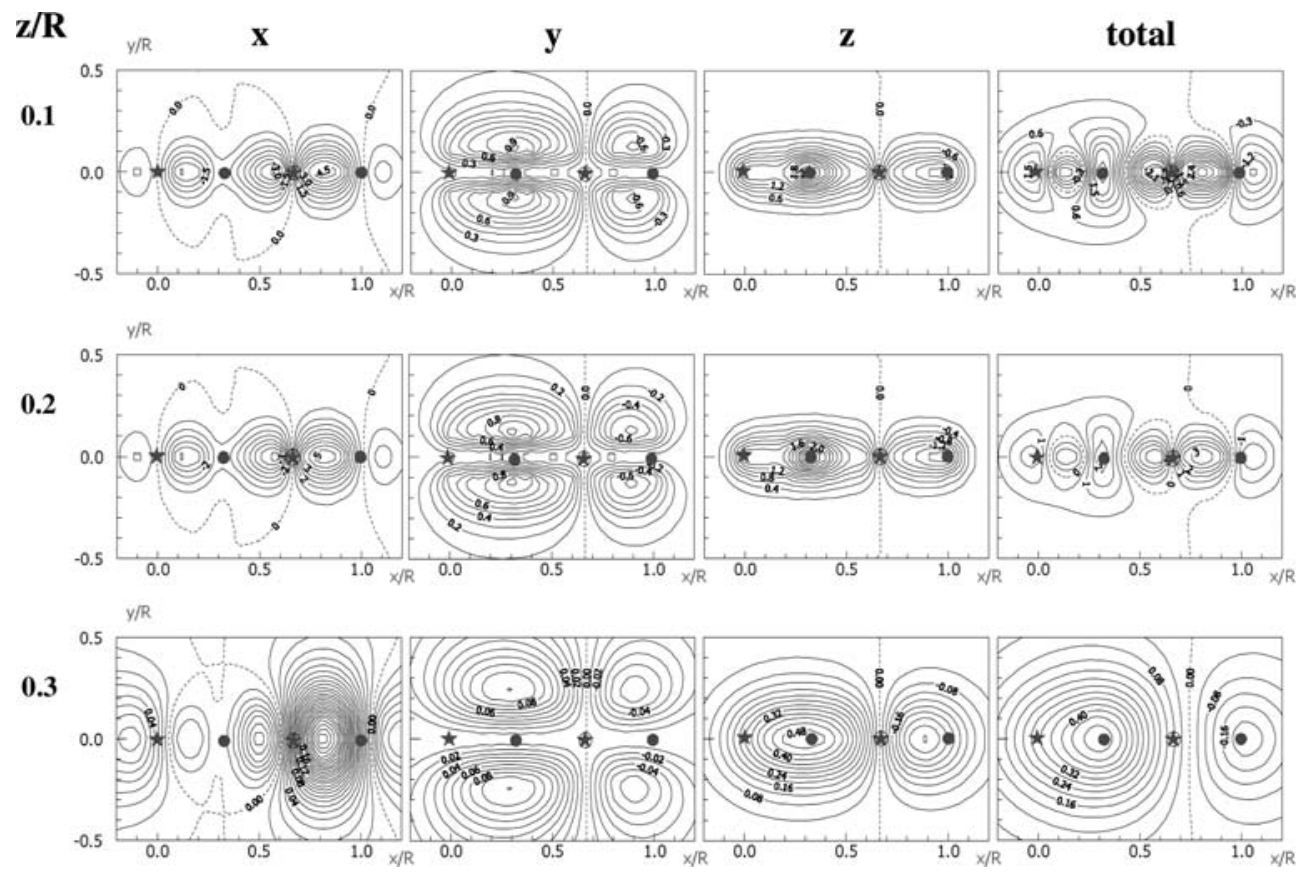

Fig. 10. Parameter-sensitivity map series of the unipole- $\gamma$ array (No. 24 in Table I). Notations are the same as in Fig. 1

Acta Geod. Geoph. Hung. 43, 2008 
We are convinced, it is possible to bring together closer these two parameter sensitivity approaches, since our method allows computing not only the response due to an elementary cube, but also the response due to larger bodies composed of the basic element. Such a study can be and should be done in the future.

\section{Acknowledgements}

Hungarian National Scientific Research Fund (projects K49604 and NI 61013); Bolyai János Research Scholarship of the Hungarian Academy of Sciences (Sándor Szalai); Ákos Gyulai and an anonymous referee, for their valuable comments.

\section{References}

Gyulai 1989: Geophysical Transactions, 35, 209-225.

Hursán G 1996: Magyar Geofizika, 37, No. 2, 106-117 (in Hungarian).

Szalai S, Szarka L 2000: Geophysical Prospecting, 48, 871-885.

Szalai S, Szarka L 2008: Acta Geod. Geoph. Hung. (present issue)

Szalai S, Szarka L, Prácser E, Bosch F, Müller I, Turberg P 2002: Geophysics, 67, 1769-1778.

Tarkhov A G 1957: Bull. Izv. Akad. Sc. USSR, 8, 11. 